\title{
Article \\ Real Color Model of a Cadaver for Deep Brain Stimulation of the Subthalamic Nucleus
}

\author{
Chung-Yoh Kim ${ }^{1}\left(\mathbb{D}\right.$, Jin-Seo Park ${ }^{1, *} \mathbb{1}$ and Beom-Sun Chung ${ }^{2, *}$ \\ 1 Department of Anatomy, School of Medicine, Dongguk University, Gyeongju 38066, Korea; \\ cykim@dongguk.edu \\ 2 Department of Anatomy, Wonju College of Medicine, Yonsei University, Wonju 26426, Korea \\ * Correspondence: park93@dongguk.ac.kr (J.-S.P.); bschung@yonsei.ac.kr (B.-S.C.)
}

Citation: Kim, C.-Y.; Park, J.-S.; Chung, B.-S. Real Color Model of a Cadaver for Deep Brain Stimulation of the Subthalamic Nucleus. Appl. Sci. 2021, 11, 4999. https://doi.org/ 10.3390/app11114999

Academic Editor: Hidenao Fukuyama

Received: 11 May 2021

Accepted: 26 May 2021

Published: 28 May 2021

Publisher's Note: MDPI stays neutral with regard to jurisdictional claims in published maps and institutional affiliations.

Copyright: (c) 2021 by the authors. Licensee MDPI, Basel, Switzerland. This article is an open access article distributed under the terms and conditions of the Creative Commons Attribution (CC BY) license (https:// creativecommons.org/licenses/by/ $4.0 /)$.

\begin{abstract}
When performing deep brain stimulation (DBS) of the subthalamic nucleus, practitioners should interpret the magnetic resonance images (MRI) correctly so they can place the DBS electrode accurately at the target without damaging the other structures. The aim of this study is to provide a real color volume model of a cadaver head that would help medical students and practitioners to better understand the sectional anatomy of DBS surgery. Sectioned images of a cadaver head were reconstructed into a real color volume model with a voxel size of $0.5 \mathrm{~mm} \times 0.5 \mathrm{~mm} \times 0.5 \mathrm{~mm}$. According to preoperative MRIs and postoperative computed tomographys (CT) of 31 patients, a virtual DBS electrode was rendered on the volume model of a cadaver. The volume model was sectioned at the classical and oblique planes to produce real color images. In addition, segmented images of a cadaver head were formed into volume models. On the classical and oblique planes, the anatomical structures around the course of the DBS electrode were identified. The entry point, waypoint, target point, and nearby structures where the DBS electrode could be misplaced were also elucidated. The oblique planes could be understood concretely by comparing the volume model of the sectioned images with that of the segmented images. The real color and high resolution of the volume model enabled observations of minute structures even on the oblique planes. The volume models can be downloaded by users to be correlated with other patients' data for grasping the anatomical orientation.
\end{abstract}

Keywords: cross-sectional anatomy; deep brain stimulation; magnetic resonance imaging; subthalamic nucleus; visible human projects

\section{Introduction}

Deep brain stimulation (DBS) of the subthalamic nucleus is used for treating Parkinson's disease and essential tremor [1,2]. In DBS surgery, the neurosurgeon navigates from the skin to the target structures of the deep brain (e.g., the subthalamic nucleus) based on magnetic resonance images (MRIs) in a probe's eye (vertical plane on a DBS electrode) and trajectory (parallel plane on the DBS electrode) planes with classical (horizontal, coronal, and sagittal) planes. On the navigation planes, they need to assess not only the overall progress of electrode insertion but also the spatial relationship between the electrode tip and nearby anatomical structures [3-6]. On the other hand, owing to the small size of the target structures for the DBS, even a minute misplacement of the electrode can have adverse effects [7]. Moreover, perforation of the lateral ventricle [8] or hemorrhaging [9] can occur during DBS surgery. Therefore, for successful DBS surgery, the practitioners have to be well informed of the anatomy of the deep brain.

However, it is difficult for undergraduate medical students to comprehend DBS surgery because of the complexity of the deep brain's anatomy. For example, identifying subthalamic nuclei and their surrounding structures in horizontal, coronal, and sagittal planes could not be easy for medical students. Even though trained neurosurgeons are 
well informed of the basic anatomy around the subthalamic nucleus, the probe's eye and trajectory planes for DBS surgery can be difficult to understand intuitively.

The existing methods to learn the deep brain's anatomy for DBS surgery have shortfalls. In the case of a conventional cadaveric dissection, it is impossible to observe diverse sectional planes at narrow intervals [10]. The subcortical atlases in the Montreal Neurological Institute space that were made from MRIs and histological slides cannot show the real color of the brain [11,12]. Recently, 7-tesla MRIs of patients were employed to depict the anatomical structures regarding DBS surgery [13]. Even though the resolution of the MRI is improved, images with real color and a high resolution can help novices to interpret an oblique MRI. Histological slides are too small to observe gross structures that the neurosurgeon needs to observe in real surgery. Therefore, an alternative method that can yield diverse sectional planes, the real color of the brain, and a high resolution is needed.

The aim of this study was to present a real color volume model which can help medical students learn the sectional anatomy of DBS surgery. In this study, from true color sectioned images of a human head [14], volume models were made. From the volume models, images of the probe's eye and trajectory planes with classical planes were reproduced. Retrospective comparison between the color images and the images of the patients who underwent the DBS surgery can help novices to understand the oblique planes of the brain and interpret patient images. Furthermore, even experienced neurosurgeons can obtain ideas for novel surgical methods by observing oblique cadaver images which differ from other sources. This study is expected to add detailed and a realistic anatomical interpretation to DBS surgery that has been improved over the course of decades.

\section{Methods}

The entire process for this study was approved by the institution review board of Ajou University Hospital in Korea (AJIRB-MED-MDB-18-315).

In a previous study, the sectioned images of a male head (interval: $0.1 \mathrm{~mm}$; pixel size: $0.1 \mathrm{~mm} \times 0.1 \mathrm{~mm}$; color depth: 48 bits of color; file format: TIFF) were produced with the use of cryomacrotome [14]. The segmented images of 294 structures (interval: $0.5 \mathrm{~mm}$; pixel size: $0.1 \mathrm{~mm}$; color depth: 48 bits of color) were produced manually [15]. The sectioned images and the segmented images of a small size can be downloaded online at neuroanatomy.kr, and the full-size images can be acquired directly from the authors.

To produce a volume model that could be handled by a personal computer, the voxel size (intervals $\times$ pixel size) of the sectioned images and segmented images was adjusted to $0.5 \mathrm{~mm} \times 0.5 \mathrm{~mm} \times 0.5 \mathrm{~mm}$ and converted to the DICOM format in Photoshop. The images of the DICOM format were reconstructed into a real color volume model and a segmented volume model. They were saved as neuroimaging informatics technology initiative (NIFTI) files using an MRIcroGL, which is MRI processing freeware available in the general personal computer environment (www.mccauslandcenter.sc.edu/mricrogl/ home, accessed on 28 May 2021) [16,17]. With this volume model, real color head structures could be observed in the arbitrary plane.

For the comparison with the clinical data, 31 patients who were diagnosed with Parkinson's disease and underwent DBS surgery in Ajou University Hospital in Korea were employed for the study (16 male, 15 female). The age ranged from 31 to 76 years (mean: 62.3; standard deviation: 10.1), and the disease duration ranged from 3 to 19 years (mean: 8.9; standard deviation: 4.0). All the DBS surgeries targeted the bilateral subthalamic nucleus and used head frame and microelectrode recording, but only the left side was chosen for this study. The patient data could be obtained thanks to the help of Professor Young Hwan Ahn of the Department of Neurosurgery at the Ajou University School of Medicine.

Preoperative MRIs (method: T1; field strength: 3 teslas; repetition time: $2500 \mathrm{~ms}$; echo time: $8 \mathrm{~ms}$; resolution: $224 \times 224$ ) and postoperative CTs (Philips Healthcare, 16 detectors; $512 \times 512$ matrix; bone series; spiral protocol; slice thickness: $2 \mathrm{~mm}$ ) of the patients were 
obtained. On the MRIs, the brain structures were visible, whereas the electrode was not visible. On the CTs, the brain structures were not visible, whereas the electrode was visible. Therefore, the CT was registered to the MRI using Stealth VR (Medtronic plc, Ireland) to obtain the coordinates of the entry point and target point in the MRI.

In the 31 patients, the entry point was the crown of the rostral middle frontal gyrus underneath the frontal bone. The target point was the dorsolateral part of the subthalamic nucleus. The entry and target points were indicated on the sectioned images so as to produce a virtual DBS electrode on the volume model (Figure 1).

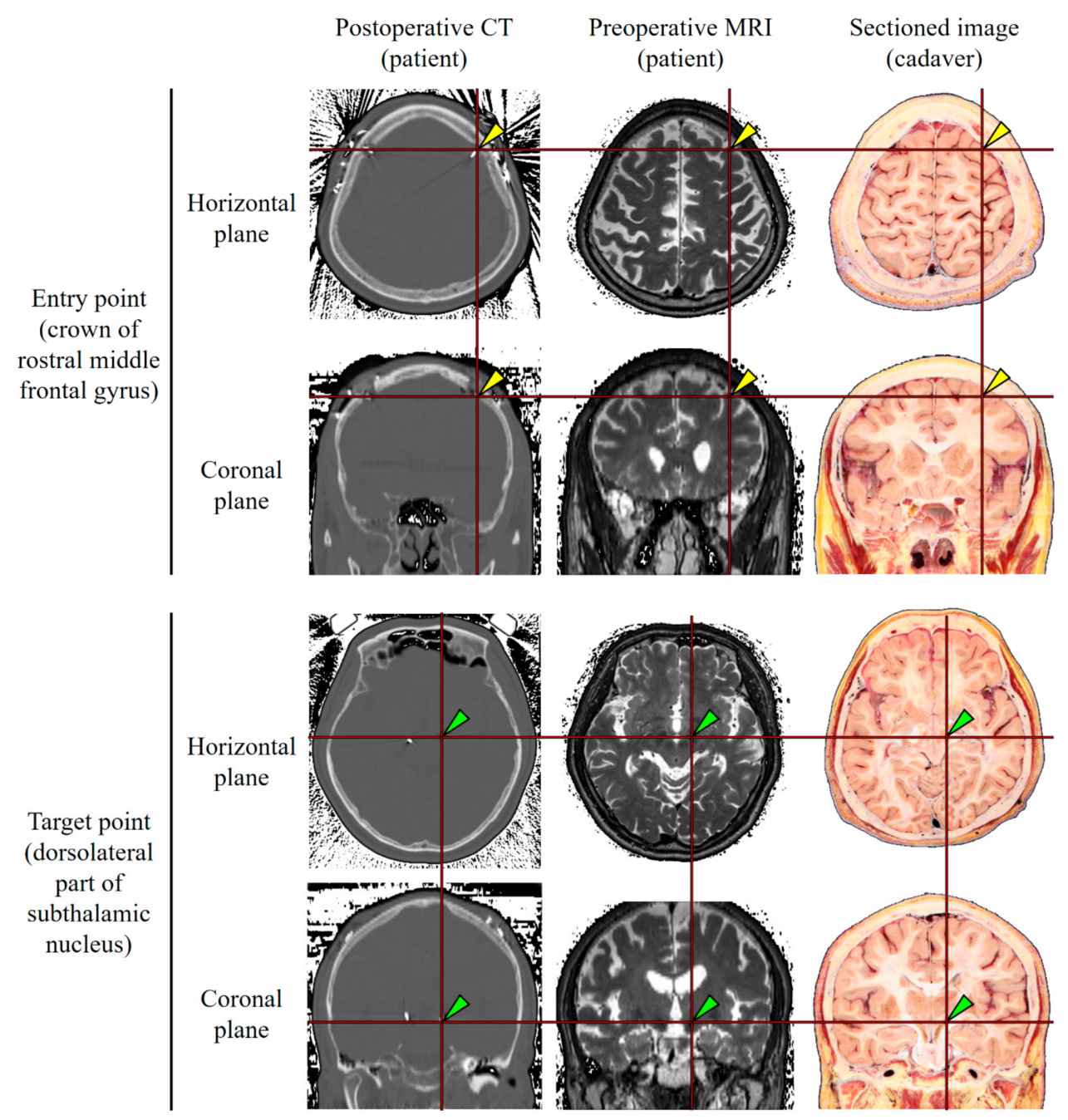

Figure 1. Entry point (yellow arrow head) and target point (green arrow head) of DBS. The entry point and target point acquired from the post-operative CT of a DBS patient as a representative were applied to the pre-operative MRI of a DBS patient through registration. The entry point and target point on the pre-operative MRI of a DBS patient were applied to the sectioned images of a cadaver by comparing the anatomy of the brain structures.

The coordinates of the entry point and target point were determined on the sectioned images based on the registration using the FSL nonlinear registration tool (FLIRT) of the FMRIB software library (FSL), which is freeware available in the general personal computer environment [18]. The registration detail was affine (12 parameter model) with linear interpolation.

Considering the three-dimensional slope of the electrode, every point between the entry point and target point were calculated using Excel 2016 (Microsoft Corp., WA) and drawn using the Extend Script Toolkit of Photoshop CS6 (Adobe System Inc., CA). Two- 
dimensional images of the electrode were made into a volume model of a virtual electrode and loaded as an overlay on the MRIcroGL.

The volume model of the cadaver head along with the overlay of the virtual electrode was loaded on the MRIcroGL. In the software, the azimuth and elevation were adjusted appropriately to determine the probe's eye and trajectory planes according to the virtual electrode. In the software, the depth of the plane was adjusted continuously at $2.3 \mathrm{~mm}$ intervals using the clip script command. Using the savebmp script command, the obliquely sectioned volume model was saved as a bitmap file where the anatomical structures could be labeled.

To distribute the volume model effectively and widely, the freeware or commonly used software in this study were selected for users to access easily and for use in the general personal computer environment.

\section{Results}

A real color volume model (file size: $98 \mathrm{MBytes),} \mathrm{segmented} \mathrm{volume} \mathrm{model} \mathrm{(file} \mathrm{size:}$ 33 MBytes), and virtual electrode (file size: 228 KBytes) were produced (voxel size: $0.5 \mathrm{~mm}$ $\times 0.5 \mathrm{~mm} \times 0.5 \mathrm{~mm}$ ). By using the real color volume model on the MRIcroGL, arbitrary planes could be produced freely. The location of the electrode could be assessed in the probe's eye planes and the horizontal, coronal, and sagittal planes. In the trajectory planes, the overall progress of electrode insertion could be assessed (Figure 2).
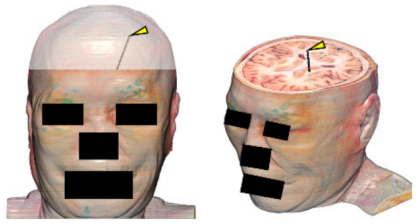

Horizontal plane
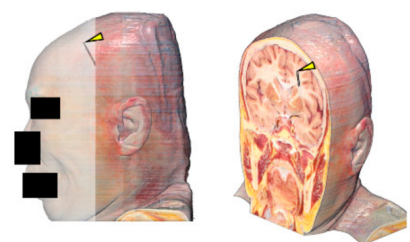

Coronal plane
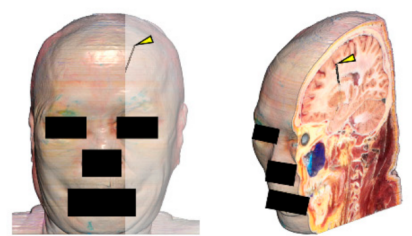

Sagittal plane
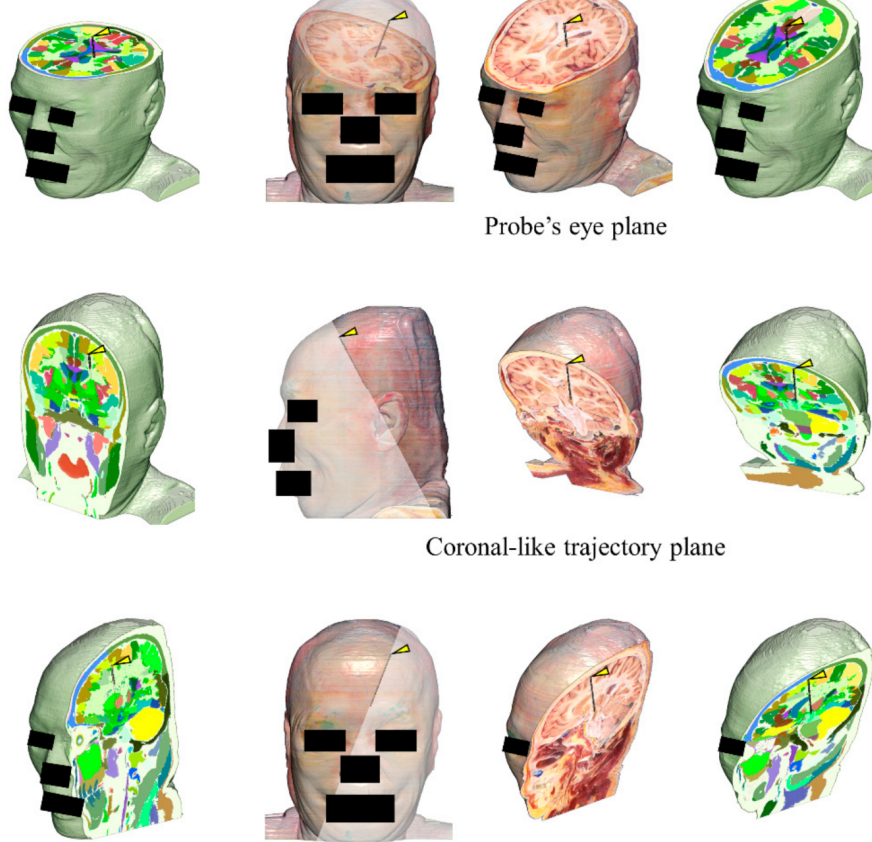

Sagittal-like trajectory plane

Figure 2. Various planes produced from the real volume model of sectioned images and a segmented volume model of a cadaver. The DBS electrode for the left subthalamic nucleus is displayed as an overlay. The entry point of the electrode is indicated with a yellow arrow head.

The entry point (crown of the rostral middle frontal gyrus) and target point (dorsolateral part of the left subthalamic nucleus) obtained from 31 patients' MRIs were marked on the horizontal, coronal, and sagittal planes on the real color volume model through the registration. On average, the entry point was located at $+50.5 \mathrm{~mm}$ in the horizontal plane, $+26.0 \mathrm{~mm}$ in the coronal plane, and $+32.0 \mathrm{~mm}$ in the sagittal plane on the anterior commissure-posterior commissure (AC-PC) coordinates. Around the entry point, the superior frontal sulcus, where surgical accidents are likely to occur, could be seen. The target point was located at $-6.5 \mathrm{~mm}$ in the horizontal plane, $-4.5 \mathrm{~mm}$ in the coronal plane, 
and $+10.0 \mathrm{~mm}$ in the sagittal plane on the AC-PC coordinates. Around the target point, care should be taken when passing the optic tract and posterior cerebral artery. The entry and target points corresponded well with the postoperative CTs of the 31 patients (Figure 3).
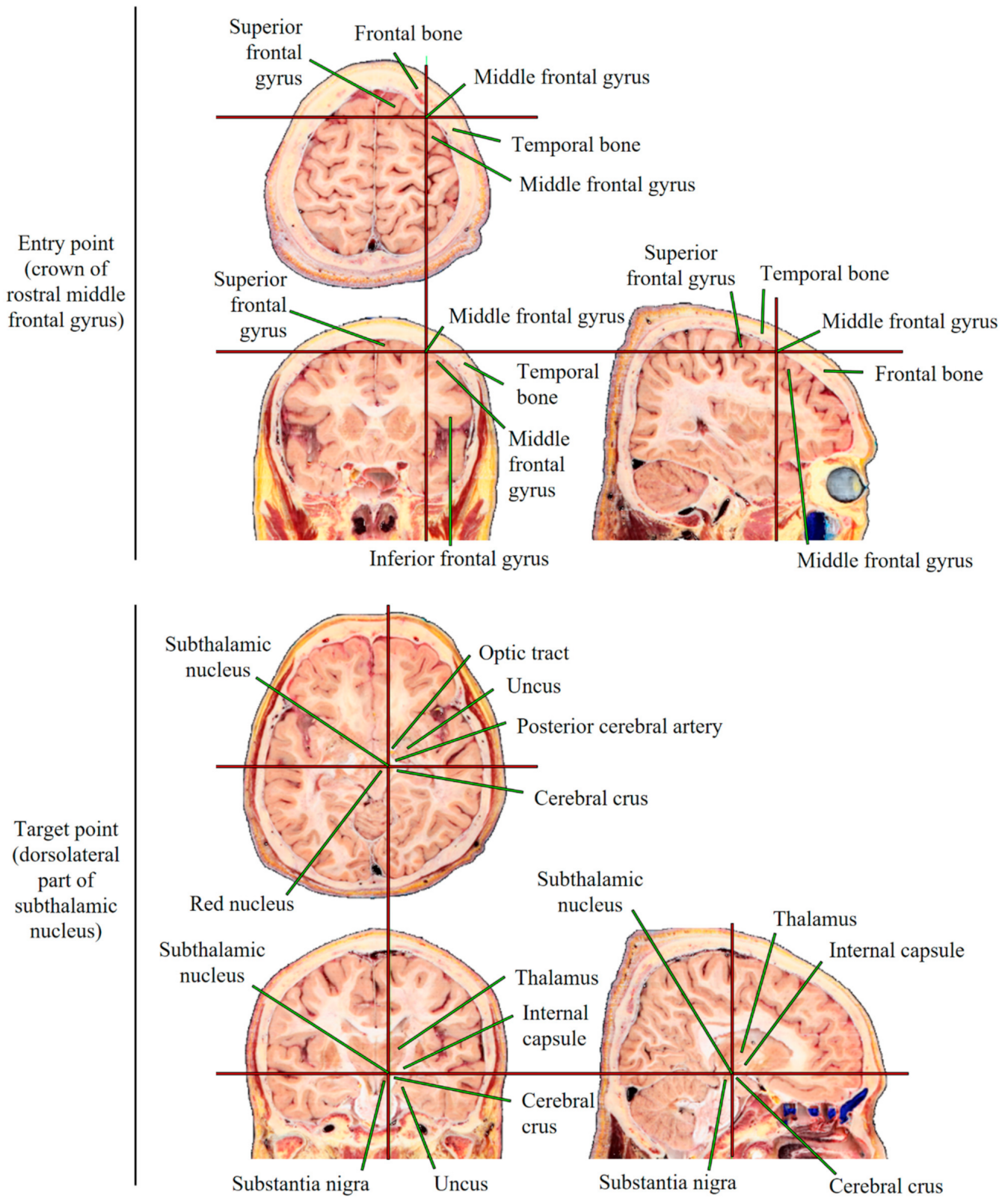

Figure 3. Horizontal, coronal, and sagittal planes on a real color volume model with the marked entry point and target point of the DBS electrode.

Along the course of the electrode in the probe's eye plane on the real color volume model, three regions where surgical accidents are likely to occur were examined: the entry point, the lateral ventricle, and the target point (subthalamic nucleus).

To avoid the artery inside the sulci in the entry point, the practitioner should choose the crown of the middle frontal gyrus. The superior and inferior frontal sulci should not be included in the course of the electrode (Figure 4) $[19,20]$. 


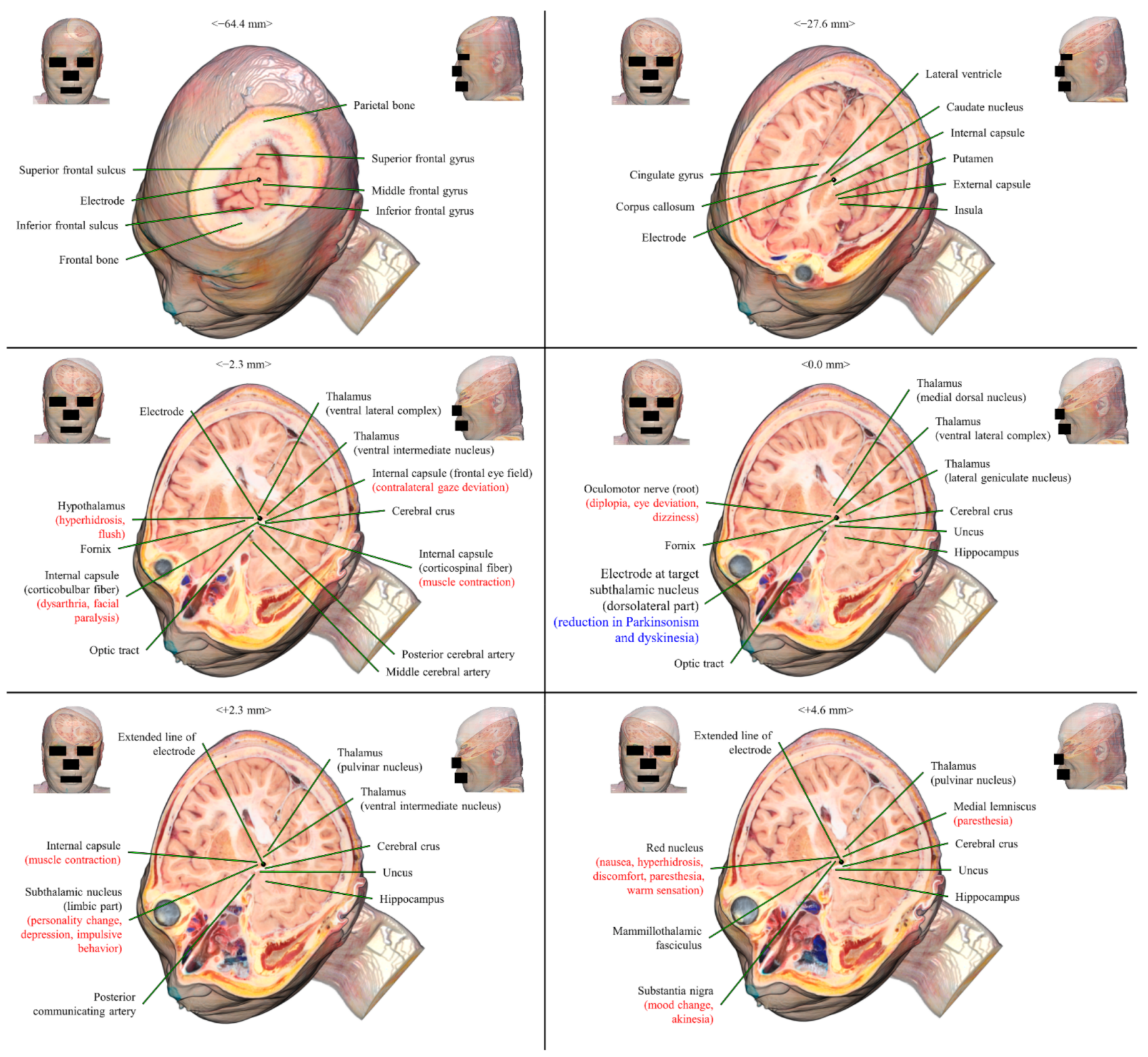

Figure 4. Probe's eye planes on a real color volume model from the entry point to the target point through the side of the lateral ventricle. The probe's eye planes are displayed from the entry point (middle frontal gyrus) to the target point (dorsolateral part of the subthalamic nucleus). The curative effects when the electrode is placed at the subthalamic nucleus are written in blue. The adverse effects when the electrode is placed at different structures are written in red.

To prevent puncturing the lateral ventricle, the electrode was passed laterally to the caudate nucleus and medially to the internal capsule. Puncturing the lateral ventricle may cause leakage of the cerebrospinal fluid (Figure 4) [21,22].

The target point on the probe's eye plane was the dorsolateral part of the subthalamic nucleus. The neighboring structures of the target point were the hypothalamus, internal capsule, oculomotor nerve, limbic part of the subthalamic nucleus, red nucleus, medial lemniscus, and substantia nigra, which were identified (Figure 3). The structures near the target were affected by the electrical stimulation rather than mechanically by the electrode [3-6].

In the coronal-like trajectory plane and sagittal-like trajectory plane on the real color volume model, the whole electrode and its neighboring structures could be seen at a glance. The electrode departed the crown of the middle frontal gyrus (entry point) and arrived 
at the dorsolateral part of the subthalamic nucleus (target point) through the lateral side of the lateral ventricle (waypoint). In the entry point, care should be taken when passing through the waypoint, target point, superior and inferior frontal sulci, lateral ventricle, and optic nerve with the probe's plane (Figure 5).
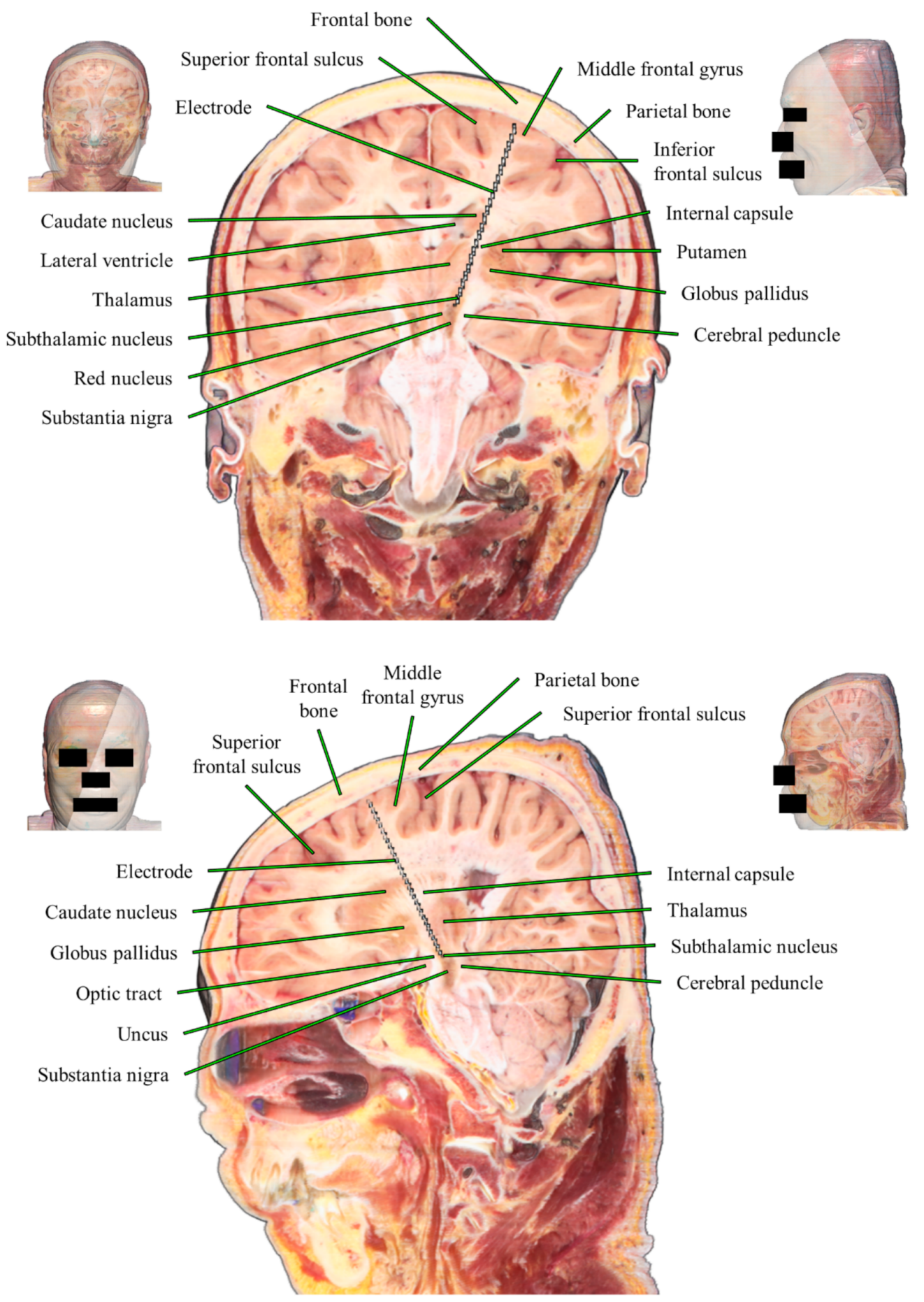

Figure 5. Trajectory planes on a real color volume model, including the whole electrode. In a coronallike trajectory plane, from the entry point to the target point, the electrode avoids the superior and inferior frontal sulci, lateral ventricle, and optic nerve (top). In a sagittal-like trajectory plane, from the entry point to the target point, the electrode departs the crown of the middle frontal gyrus and arrives at dorsolateral part of the subthalamic nucleus (bottom). 


\section{Discussion}

The real color volume model of this study is the convergence between an actual cadaver dissection and MRI processing. The strong points of the real color volume model for DBS surgery are as follows.

The real color volume model enables observations of minute anatomical structures. It can be difficult for medical students to identify deep brain structures in gray scale images of an MRI or CT. By virtue of the real color of the volume model, medical students can be familiar with the sectional brain's anatomy before they study DBS surgery. In the case of neurosurgeons performing DBS surgery, misplacement of the DBS electrode on the medial lemniscus or substantia nigra can result in various adverse effects. Even within the subthalamic nucleus, its motor, limbic, and associative subdivisions have different functions [23,24]. Therefore, neurosurgeons interpret preoperative MRIs of patients to plan the DBS approach and perform an actual cadaver dissection to grasp the detailed anatomy of the deep brain [10]. These minute structures of the deep brain can be observed on the real color volume model, owing to the voxel size of $0.5 \mathrm{~mm} \times 0.5 \mathrm{~mm} \times 0.5 \mathrm{~mm}$. The real color volume can be helpful in associating the actual cadaver to medical images of patients (Figure 1).

The volume model of the segmentation data is helpful in learning the sectional anatomy. For example, it is difficult for medical students to identify the sulci and gyri of the cerebral cortex on the horizontal, coronal, and sagittal planes. Even for experts, identifying the sulci and gyri on the oblique planes is not easy. Owing to the head folding during development, structures derived from the telencephalon have a complicated spatial relationship. The cerebral cortex and deep brain structures can be identified by sectioning the volume model of the segmentation data (Figure 2).

The volume model can be correlated to the data of other trajectories for DBS surgery. In this study, the averages of the coordinates from 31 patients were adopted to determine the trajectory of the electrode. Since the coordinates were based on the clinical data, the entry points and target points were determined by minimizing the potential adverse effects of electrode insertion. If the coordinates of the entry and target points of other DBS trajectories are adopted, the related anatomical structures can be displayed on the real color volume model of this study (Figures 3-5). By analyzing the structures along the trajectory of the electrode, potential adverse effects of the other DBS trajectories can be discovered. Through this trial, the model may propose new ideas for improved trajectories in DBS surgery for neurosurgeons.

The limitation of this study was that the volume model was produced based on a single cadaver, although it had no pathological finding in the brain [14]. Deep brain structures can individually vary according to the size of the head and brain. Therefore, the users should keep in mind that the images of a single cadaver cannot represent the anatomical variations of individual patients. The standardized brain anatomy can be complemented by the existing coordination system and registration algorithm, which also can be obtained gratis $[18,25]$.

DBS of the subthalamic nucleus requires neurosurgeons to target small-sized structures precisely because minute misplacement of the electrode can have adverse effects. The real color volume model of this study enables users to identify detailed anatomical structures not only in classical planes but also in oblique planes. The volume model can be helpful for students or novice neurosurgeons to study the anatomy of the deep brain and plan DBS surgery thanks to the real color. Furthermore, the model may propose ideas to find improved approaches in DBS surgery for neurosurgeons by observing arbitrary oblique planes.

\section{Conclusions}

The real color volume models of this study are accompanied by the following supplementary software. Surface models made from the same cadaver head can be used to help learn the stereoscopic anatomy [15]. The volume model of the cadaver has been 
peeled from the skin to elucidate the surface anatomy in a higher resolution [26]. The browsing software of the horizontal sectioned images along with the segmentation data can be operated with Android mobile devices [27]. The real color volume model, compared with DBS patient images, will function as an effective tool for medical students to learn sectional anatomy for DBS surgery. The data from this study will aid in the assessment of DBS surgery, like the three-dimensional (3D) digital atlas. To help neurosurgeons read MRIs for performing DBS surgery, the real color volume data and tools for performing virtual DBS surgery have been distributed free of charge from the author's homepage. In a future study, a prospective comparison with the patients' medical images, treatment results, and the cadaver volume model can be attempted for clinical validation. In addition, DBS has been performed experimentally on non-human primates [28]. The method of this study can be applied to a real volume model made from a rhesus monkey produced by the authors [29].

Author Contributions: Conceptualization, J.-S.P. and B.-S.C.; methodology, B.-S.C.; software, C.-Y.K. and B.-S.C.; validation, J.-S.P.; formal analysis, B.-S.C.; investigation, C.-Y.K.; writing-original draft preparation, J.-S.P.; writing-review and editing, C.-Y.K. and B.-S.C.; supervision, J.-S.P.; funding acquisition, J.-S.P. All authors have read and agreed to the published version of the manuscript.

Funding: This work was supported by the National Research Foundation of Korea (NRF) grant funded by the Korean government (MSIT) (No. 2019R1F1A1059842).

Institutional Review Board Statement: The entire process for this study was approved by the institution review board of Ajou University Hospital in Korea (AJIRB-MED-MDB-18-315).

Informed Consent Statement: Not applicable.

Data Availability Statement: Data are contained within the article.

Acknowledgments: The authors gratefully thank Ajou University Hospital in Korea for their assistance.

Conflicts of Interest: The authors declare no conflict of interest.

\section{References}

1. Deuschl, G.; Schade-Brittinger, C.; Krack, P.; Volkmann, J.; Schäfer, H.; Bötzel, K.; Daniels, C.; Deutschländer, A.; Dillmann, U.; Eisner, W.; et al. A randomized trial of deep-brain stimulation for Parkinson's disease. N. Eng. J. Med. 2006, 355, 896-908. [CrossRef]

2. Benabid, A.L.; Chabardes, S.; Mitrofanis, J.; Pollak, P. Deep brain stimulation of the subthalamic nucleus for the treatment of Parkinson's disease. Lancet Neurol. 2009, 8, 67-81. [CrossRef]

3. Tarsy, D.; Norregaard, T.; Hubble, J. Surgical Treatment of Parkinson's Disease and Other Movement Disorders; Springer Science \& Business Media: Berlin, Germany, 2002; p. 153.

4. Castro, F.S.; Pollo, C.; Meuli, R.; Maeder, P.; Cuisenaire, O.; Cuadra, M.B.; Villemure, J.G.; Thiran, J.P. A cross validation study of deep brain stimulation targeting: From experts to atlas-based, segmentation-based and automatic registration algorithms. IEEE Trans. Med. Imaging 2006, 25, 1440-1450. [CrossRef]

5. Baltuch, G.H.; Stern, M.B. (Eds.) Deep Brain Stimulation for Parkinson's Disease; CRC Press: Boca Raton, FL, USA, 2007.

6. Wang, D.; Sanchez, J.; Foote, K.D.; Sudhyadhom, A.; Bhatti, M.T.; Lewis, S.; Okun, M.S. Failed DBS for palliation of visual problems in a case of oculopalatal tremor. Parkinsonism Relat. Disord. 2009, 15, 71-73. [CrossRef]

7. Kleiner-Fisman, G.; Herzog, J.; Fisman, D.N.; Tamma, F.; Lyons, K.E.; Pahwa, R.; Lang, A.E.; Deuschl, G. Subthalamic nucleus deep brain stimulation: Summary and meta-analysis of outcomes. Mov. Disord. 2006, 21, S290-S304. [CrossRef]

8. Zrinzo, L.; van Hulzen, A.L.; Gorgulho, A.A.; Limousin, P.; Staal, M.J.; De Salles, A.A.; Hariz, M.I. Avoiding the ventricle: A simple step to improve accuracy of anatomical targeting during deep brain stimulation. J. Neurosurg. 2009, 110, 1283-1290. [CrossRef] [PubMed]

9. Ben-Haim, S.; Asaad, W.F.; Gale, J.T.; Eskandar, E.N. Risk factors for hemorrhage during microelectrode-guided deep brain stimulation and the introduction of an improved microelectrode design. Neurosurgery 2009, 64, 754-763. [CrossRef] [PubMed]

10. Mavridis, I.N.; Michalinos, A.; Anagnostopoulou, S. Deep brain stimulation's anatomy: Step by step evaluation of the extracerebral surgical technique. OA Anat. 2013, 1, 5. [CrossRef]

11. Chakravarty, M.M.; Bertrand, G.; Hodge, C.P.; Sadikot, A.F.; Collins, D.L. The creation of a brain atlas for image guided neurosurgery using serial histological data. Neuroimage 2006, 30, 359-376. [CrossRef] 
12. Ewert, S.; Plettig, P.; Li, N.; Chakravarty, M.M.; Collins, D.L.; Herrington, T.M.; Kühn, A.A.; Horn, A. Toward defining deep brain stimulation targets in MNI space: A subcortical atlas based on multimodal MRI, histology and structural connectivity. Neuroimage 2018, 170, 271-282. [CrossRef]

13. Duchin, Y.; Shamir, R.R.; Patriat, R.; Kim, J.; Vitek, J.L.; Sapiro, G.; Harel, N. Patient-specific anatomical model for deep brain stimulation based on 7 Tesla MRI. PLoS ONE 2018, 13, e0201469. [CrossRef]

14. Park, J.S.; Chung, M.S.; Shin, D.S.; Har, D.H.; Cho, Z.H.; Kim, Y.B.; Han, J.Y.; Chi, J.G. Sectioned images of the cadaver head including the brain and correspondences with ultrahigh field 7.0 T MRIs. Proc. IEEE 2009, 97, 1988-1996. [CrossRef]

15. Shin, D.S.; Jang, H.G.; Park, J.S.; Park, H.S.; Lee, S.; Chung, M.S. Accessible and informative sectioned images and surface models of a cadaver head. J. Craniofac. Surg. 2012, 23, 1176-1180. [CrossRef] [PubMed]

16. Li, X.; Morgan, P.S.; Ashburner, J.; Smith, J.; Rorden, C. The first step for neuroimaging data analysis: DICOM to NIfTI conversion. J. Neurosci. Methods 2016, 264, 47-56. [CrossRef] [PubMed]

17. Chung, B.S.; Park, J.S. Real-color volume models made from real-color sectioned images of Visible Korean. J. Korean Med. Sci. 2019, 34. [CrossRef] [PubMed]

18. Jenkinson, M.; Beckmann, C.F.; Behrens, T.E.; Woolrich, M.W.; Smith, S.M. FSL. Neuroimage 2012, 62, 782-790. [CrossRef]

19. Machado, A.; Rezai, A.R.; Kopell, B.H.; Gross, R.E.; Sharan, A.D.; Benabid, A.L. Deep brain stimulation for Parkinson's disease: Surgical technique and perioperative management. Mov. Disord. 2006, 21, S247-S258. [CrossRef]

20. Xiaowu, H.; Xiufeng, J.; Xiaoping, Z.; Bin, H.; Laixing, W.; Yiqun, C.; Jinchuan, L.; Aiguo, J.; Jianmin, L. Risks of intracranial hemorrhage in patients with Parkinson's disease receiving deep brain stimulation and ablation. Parkinsonism Relat. Disord. 2010, 16, 96-100. [CrossRef]

21. Oh, M.Y.; Abosch, A.; Kim, S.H.; Lang, A.E.; Lozano, A.M. Long-term hardware-related complications of deep brain stimulation. Neurosurgery 2002, 50, 1268-1276. [CrossRef]

22. Bittar, R.G.; Otero, S.; Carter, H.; Aziz, T.Z. Deep brain stimulation for phantom limb pain. J. Clin. Neurosci. 2005, 12, 399-404. [CrossRef]

23. Benarroch, E.E. Subthalamic nucleus and its connections: Anatomic substrate for the network effects of deep brain stimulation. Neurology 2008, 70, 1991-1995. [CrossRef] [PubMed]

24. Clement Hamani, G.F.; Heinsen, H.; Plantinga, B.R.; Temel, Y.; Uludag, K.; Alho, E.; Teixeira, M.J.; Amaro, E.; Fonoff, E.T. Subthalamic nucleus deep brain stimulation: Basic concepts and novel perspectives. Eneuro 2017, 4. [CrossRef]

25. Evans, A.C.; Janke, A.L.; Collins, D.L.; Baillet, S. Brain templates and atlases. Neuroimage 2012, 62, 911-922. [CrossRef] [PubMed]

26. Kwon, K.; Shin, H.K.; Shin, B.S.; Park, J.S. Serially peeled images of the curved surface of the face based on cross-sectional images for use in plastic surgery. J. Plast. Reconstr. Aesthet. Surg. 2016, 69, 727-729. [CrossRef] [PubMed]

27. Lee, S.B.; Chung, B.S.; Chung, M.S.; Youn, C.; Park, J.S. Browsing Software of the Head Sectioned Images for the Android Mobile Device. Int. J. Morphol. 2017, 35, 1337-1382. [CrossRef]

28. Miocinovic, S.; Zhang, J.; Xu, W.; Russo, G.S.; Vitek, J.L.; McIntyre, C.C. Stereotactic neurosurgical planning, recording, and visualization for deep brain stimulation in non-human primates. J. Neurosci. Methods 2007, 162, 32-41. [CrossRef]

29. Chung, B.S.; Jeon, C.Y.; Huh, J.W.; Jeong, K.J.; Har, D.; Kwack, K.S.; Park, J.S. Rise of the visible monkey: Sectioned images of rhesus monkey. J. Korean Med. Sci. 2019, 34. [CrossRef] [PubMed] 